\title{
Characterizations of the cycle-complete and folk solutions for minimum cost spanning tree problems
}

\author{
Christian Trudeau (University of Windsor)
}

Working paper 13 - 03

Working papers are in draft form. This working paper is distributed for purposes of comment and discussion only. It may not be reproduced without permission of the copyright holder. Copies of working papers are available from the author or at http://ideas.repec.org/s/wis/wpaper.html. 


\title{
Characterizations of the cycle-complete and folk solutions for minimum cost spanning tree problems
}

\author{
Christian Trudeau*
}

This draft: April 2013

\begin{abstract}
Minimum cost spanning tree problems connect agents efficiently to a source when agents are located at different points and the cost of using an edge is fixed. The folk and cycle-complete cost sharing solutions always offer core allocations. We provide similar characterizations for both. A new property is based on the following observation: when all agents have the same cost to connect to the source, we can connect one of them to the source then connect all other agents to him, as if he was the source. Cost sharing should also be done in these two steps. We also use some common properties: Core Selection, Piecewise Linearity and an independence property.

The solutions are differentiated by properties that apply when the cheapest edge to the source gets cheaper. Either the savings are equally distributed among all agents (folk) or the agent on that edge gets all of the savings (cycle-complete).

Keywords: Minimum cost spanning tree problems; folk solution; cycle-complete solution; core.

JEL Classifications: C71, D63
\end{abstract}

\section{Introduction}

Minimum cost spanning tree (mcst) problems model a situation where agents are located at different points and need to be connected to a source in order to obtain a good or information. Agents do not care if they are connected directly to the source or indirectly through other agents who are. The cost to build a link between two agents or an agent and the source is a fixed number, meaning that the cost is the same whether one or ten agents use that particular link. Mcst problems can be used to model various real-life problems, from telephone and cable TV to water supply networks.

We are interested in the cost sharing problem related to mcst problems. Once agents decide to build the network, the common cost of construction must be split among the participants. The application of the Shapley value to this problem, first studied in Bird (1976) and known as the Kar solution (Kar (2002)), has interesting properties but might be outside of the core, meaning that some coalitions might be better off leaving the group and undertaking the project by themselves. The folk (first suggested by Feltkamp et al (1994) and rediscovered independently by Bergantinos and Vidal-Puga (2007)) and cycle-complete (Trudeau (2012b)) solutions offer similar remedies. Both modify the cost matrix before applying the Shapley value on the modified game. To obtain the modifications needed to compute the folk solution, for each pair of agents (or for each agent and the source), we find the path between them for which the most expensive edge is as cheap as possible. We then assign that cost to this pair of agents in the modified matrix. For the cycle-complete solution, we proceed in the same manner, but look at cycles instead of paths.

Adding to the similarities of the methods, this paper provides similar characterizations for both solutions. To do so, we introduce a new property called Decomposition. Suppose that all agents have

*Department of Economics, University of Windsor

401 Sunset Avenue, Windsor, Ontario, Canada. Email: trudeauc@uwindsor.ca 
the same high cost to connect to the source. Then, we can find the mcst as follows: we pick one agent randomly, and connect him to the source. The problem then becomes as if that agent was the source, as others have to connect to him, directly or indirectly. The Decomposition property states that the cost sharing can be done in the same manner. We combine this property with the well-known properties of Core Selection and Piecewise Linearity (Bogomolnaia and Moulin (2010)), as well as an independence property between groups that is similar to those used in Kar (2002), Bergantinos and Vidal-Puga (2007) and Trudeau (2013). The difference is that here it applies on branches of the minimum cost spanning tree: if $S$ connects to the source through agent $i$ and is completely independent of the other agents, we should be able to remove agents not in $S \cup\{i\}$ from the problem without affecting the cost shares of agents in $S$.

The final property deals with the following problem: suppose that all agents can connect at zero cost to agent $i$, who has the lowest cost to connect directly to the source. If the cost to connect agent $i$ drops further, how should we divide the savings? The property of Constant Share of Cost Reduction imposes that this be done in a consistent manner, with agent $i$ getting the same proportion of the savings regardless of the number of agents. This property is enough, when combined with the properties described above, to characterize the convex combination of the folk and cycle-complete solutions. There are two natural strengthenings of that property: Equal Share of Cost Reduction says that the savings should be split evenly among all agents, while Full Share of Cost Reduction states that agent $i$ should get all of it. The difference between the two properties is a difference of interpretation: does an agent have property rights over his location (i.e., can he prevent others from connecting through his location) and is an agent responsible for his location and the corresponding costs?

The characterization of the folk solution is obtained with Equal Share of Cost Reduction, while the characterization of the cycle-complete solution uses Full Share of Cost Reduction, illustrating clearly the difference in approach between the two methods. The characterization of the folk solution can be further refined by dropping the Decomposition property.

The paper is divided as follows: Section 2 defines the minimum cost spanning tree problems and the two cost sharing solutions studied in this paper. The properties used in the paper are described in Section 3. Characterization results are in Section 4. Section 5 contains some discussions. Some supporting lemmas are in Appendix.

\section{The setting}

\subsection{Minimum cost spanning tree problems}

Let $\mathcal{N}=\{1,2, \ldots\}$ be the set of potential participants and $N \subseteq \mathcal{N}$ be the set of agents that actually need to be connected to the source, denoted by 0 . Let $N_{0}=N \cup\{0\}$. For any set $Z \subseteq \mathcal{N} \cup\{0\}$, define $Z^{p}$ as the set of all non-ordered pairs $(i, j)$ of elements of $Z$. In our context, any element $(i, j)$ of $Z^{p}$ represents the edge between $i$ and $j$. Let $c=\left(c_{e}\right)_{e \in N_{0}^{p}}$ be a vector in $\mathbb{R}_{+}^{N_{0}^{p}}$ with $c_{e}$ representing the cost of edge $e$. Let $\Gamma(N)$ be the set of all cost vectors when the set of agents is $N$, with $N \subseteq \mathcal{N}$. Let $\Gamma$ be the set of all cost vectors, for all possible $N$. Since $c$ assigns cost to all edges $e$, we often abuse language and call $c$ a cost matrix. A minimum cost spanning tree problem is a triple $(0, N, c)$. Since 0 does not change, we omit it in the following and simply identify a mcst problem as $(N, c)$, with $N \subseteq \mathcal{N}$ and $c \in \Gamma(N)$.

A cycle $p_{l l}$ is a set of $K \geq 3$ edges $\left(i_{k}, i_{k+1}\right)$, with $k \in[0, K-1]$ and such that $i_{0}=i_{K}=l$ and $i_{1}, \ldots, i_{K-1}$ distinct and different than $l$. A path $p_{l m}$ between $l$ and $m$ is a set of $K$ edges $\left(i_{k}, i_{k+1}\right)$, with $k \in[0, K-1]$, containing no cycle and such that $i_{0}=l, i_{K}=m$ and $i_{1}, \ldots, i_{K-1}$ distinct and different from $l$ and $m$. Let $P_{l m}\left(N_{0}\right)$ be the set of all such paths between $l$ and $m$. For a set of edges $Y \in N_{0}^{p}$, we say that $Y$ is in $S \subseteq N_{0}$ if for all $(i, j) \in Y, i, j \in S$. We say that $Y$ contains a cycle in $S$ if, for all $i \in S$, there exists a cycle $p_{i i}$ in $S$ such that all elements of $p_{i i}$ are also in $Y$. We say that a path $p_{l m}$ is a free path if $c_{e}=0$ for all $e \in p_{l m}$. 
A spanning tree is a non-orientated graph without cycles that connects all elements of $N_{0}$. A spanning tree $t$ is identified by the set of its edges. Its associated cost is $\sum_{e \in t} c_{e}$. The spanning trees with the minimum cost are called minimum cost spanning trees (mcst). It is well known that we can find a mcst in polynomial time (Prim (1957), Kruskal (1956)). Let $C(N, c)$ be the cost of these mcst. Let $t^{*}$ be one of those mcst and $T^{*}(c)$ be the set of all mcst. For any $t^{*} \in T^{*}(c), p_{i j}\left(t^{*}\right)$ is the unique path from $i$ to $j$ in $t^{*}$. We say that $j$ is a follower of $i$ in $c$ if for all $t^{*} \in T^{*}(c)$ there is a $k_{t^{*}} \in N_{0} \backslash\{i, j\}$ such that $\left(i, k_{t^{*}}\right) \in p_{0 j}\left(t^{*}\right)$.

Let $c^{S}$ be the restriction of the cost matrix $c$ to the coalition $S_{0} \subseteq N_{0}$. Let $C(S, c)$ be the cost of the mcst of the problem $\left(S, c^{S}\right)$. Given these definitions, we say that $C$ is the stand-alone cost function associated with $c$.

\subsection{Cost sharing solutions}

For a problem $(N, c)$, a cost allocation $y \in \mathbb{R}^{N}$ assigns a cost share to each agent, and the budget balance condition is $\sum_{i \in N} y_{i}=C(N, c)$.

A cost sharing solution (or rule) assigns a cost allocation $y(N, c)$ to any admissible mcst problem $(N, c)$. We introduce the two solutions that are the focus of the paper.

From any cost matrix $c$, we can define the irreducible cost matrix $\bar{c}$ as follows:

$$
\bar{c}_{i j}=\min _{p_{i j} \in P_{i j}\left(N_{0}\right)} \max _{e \in p_{i j}} c_{e} \text { for all } i, j \in N_{0} .
$$

From any cost matrix $c$, we can define the cycle-complete cost matrix $c^{*}$ as follows:

$$
\begin{aligned}
c_{i j}^{*} & =\max _{k \in N \backslash\{i, j\}} \overline{c_{i j}^{N \backslash\{k\}}} \text { for all } i, j \in N \\
c_{0 i}^{*} & =\max _{k \in N \backslash\{i\}} \overline{c_{i j}^{N \backslash\{k\}}} \text { for all } i \in N .
\end{aligned}
$$

where $\overline{c_{i j}^{N \backslash\{k\}}}$ indicate the cost of edge $(i, j)$ on the matrix that we first restricted to agents in $N \backslash\{k\}$ before transforming into an irreducible matrix.

The cycle complete matrix can also be defined using cycles (Trudeau (2012b)): for edge $(i, j)$, we look at cycles that go through $i$ and $j$. If there is one such cycle such that its most expensive edge is cheaper than a direct connection on edge $(i, j)$, we assign this cost to edge $(i, j)$.

The folk solution is the Shapley value of $C(\cdot, \bar{c})$ while the cycle-complete solution is the Shapley value of $C\left(\cdot, c^{*}\right)$. The Kar solution $y^{K}$ is simply the Shapley value of $C(\cdot, c)$.

\section{Properties}

We use a set of properties that include some familiar ones as well as some new ones. We start by defining the new ones.

To introduce the Decomposition property, consider Figure 1a, where agents are identified in bold and other numbers are the cost of the different edges. All agents have the same cost to connect to the source, with costs to connect two agents together being smaller. An obviously optimal way to construct the mcst is to randomly select one agent to connect to the source, and to then connect everybody else to that agent. Therefore, once we have selected who to connect to the source, the problem becomes as if that agent is the source. Figure $1 \mathrm{~b}$ shows the resulting problems if we connect one of the agents to the source.

The Decomposition property goes through with that idea not only for building the mcst, but also for sharing its cost. More precisely, if $i$ was selected to be connected to the source, he pays that cost, while the cost to connect the remaining agents is computed on the reduced problem where $i$ acts as the source. Since agent $i$ pays more than others, it is not fair to him. To recover fairness, every agent takes 
Figure 1: Illustration of Decomposition

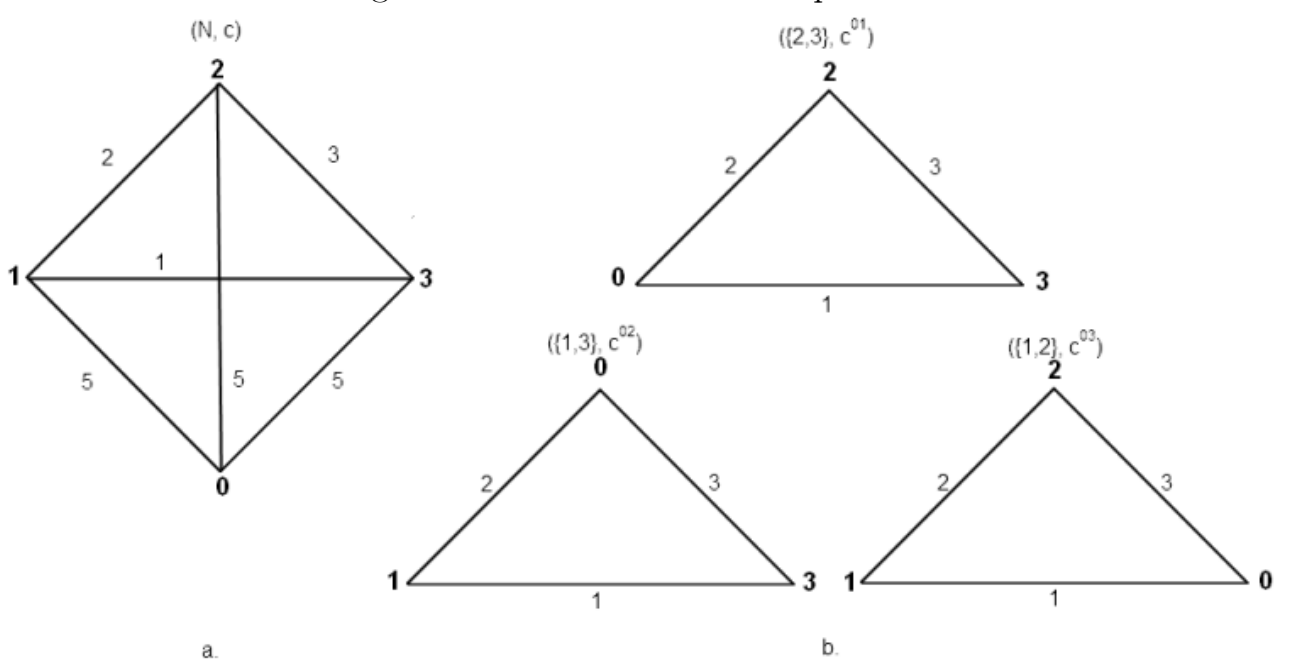

its turn being the source, and we take the average. Decomposition has a nice consistency implication, as it treats in the same manner problems where agents all have to connect to a given node, regardless of the fact that this node contains the source or an agent.

The property bears resemblance to the Problem Separation property of Trudeau (2013), which also aims to divide the connection to the source from the connection between agents. The main difference is that Problem Separation has a larger scope, applying in cases where agents have different direct connection costs to the source. There is also a different treatment of the problem of connecting agents together. The larger scope results in the cycle-complete solution failing to satisfy the Problem Separation property. While Decomposition has a limited scope, in the cases where it applies the interpretation and relevance of the process is absolutely natural.

We now formally define the property. Let $c^{0 i}$ be such that for all $j, k \in N \backslash\{i\}, c_{0 j}^{0 i}=c_{i j}$ and $c_{j k}^{0 i}=c_{j k}$. It is the cost matrix that represents the situation when we assume that agent $i$ is the source. $C\left(\cdot, c^{0 i}\right)$ is the corresponding stand-alone game, defined over the set of agents $N \backslash\{i\}$. Let $\hat{c}$ be such that for all $i \in N, \hat{c}_{0 i}=c_{0 i}$, while $\hat{c}_{i j}=0$ for all $i, j \in N$. Then, all that is left are the costs to connect agents to the source. The mcst is such that one agent is connected to the source and all others are connected to him (at no cost since $\hat{c}_{i j}=0$ for all $i, j \in N$ ).

Decomposition: For any mcst problem $(N, c)$ such that $c_{0 i}=a, c_{j k} \leq a$ for all $i, j, k \in N$, we have $y_{i}(N, c)=\sum_{j \in N \backslash\{i\}} \frac{y_{i}\left(N \backslash\{j\}, c^{0 j}\right)}{|N|}+y_{i}(N, \hat{c})$ for all $i \in N$.

Notice that if $c_{0 i}=a, c_{j k} \leq a$ for all $i, j, k \in N$ and $T \subseteq N \backslash\{i\}$ we have $C\left(T, c^{0 i}\right)=C(T \cup\{i\}, c)-a$.

Our second property is the Branch Cutting property, which is close to the Group Independence and Separability properties found in Kar (2002), Bergantinos and Vidal-Puga (2007) and Trudeau (2013). These properties state that if we can split our agents into two groups that can be connected independently to the source, then we can do the cost sharing separately on these two groups. The property we introduce also applies this independence to different branches of the mcst.

Consider Figure 2a, where (one of) the mcst is identified by the dashed lines. Agents 1 and 2 form a branch that connects independently to the source. Even more, the edges between $\{1,2\}$ and $\{3\}$ are all at least as expensive as the edges connecting these agents to the source, meaning that there are never any gains in using them. Since groups $\{1,2\}$ and $\{3\}$ have no gains to cooperate together, we might as well treat them separately. Our Branch Cutting property, just like Group Independence, says that in that case, we can remove agent 3 from the problem to compute the shares of agents 1 and 2, and vice-versa. Branch Cutting, however, implies more. Consider Figure 2b. Agents 1 and 2 still have 
Figure 2: Illustration of Branch Cutting

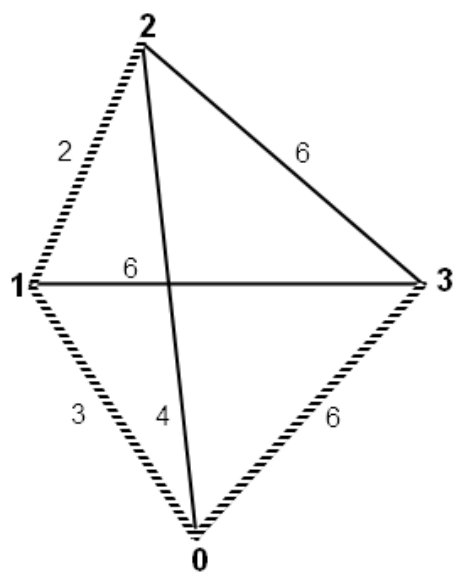

a.

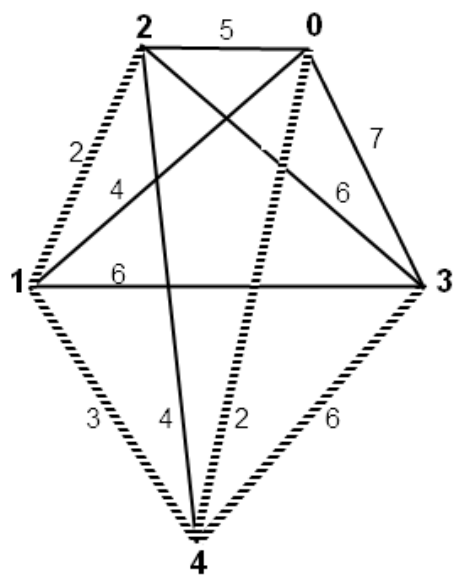

b.

no gains to cooperate with agent 3 , but now instead of connecting independently to the source, they connect independently to agent 4 , who has an advantageous connection to the source. While Group Independence says nothing about this case, we argue that this situation is no different than the one in Figure 2a, and that we should still be able to remove agent 3 to compute the shares of agents 1 and 2, and vice-versa. The Branch Cutting property does just that. Just like the name of the property alludes to, if two branches of the mcst are independent, we should be able to "cut" one of them and compute the shares of the other branch using that reduced problem. ${ }^{1}$

Suppose that the branch that we are considering connects at node $k$. We further restrict the application of this property to cases where the edges within the branch are at least as costly as the most expensive edge on an optimal path from the source to $k$. In those cases, when building the mcst, agent $k$ will be connected to the source before any member of the branch. Once $k$ is connected to the source, it remains to connect members of the branch to $k$, with these members having no interest in being connected to anybody else.

We allow $k$ to be the source; in that case, Branch Cutting is equivalent to the Group Independence property of Trudeau (2013).

We now formally define the property. To define the restricted games once we have removed some branches, we need the following notation. Since for $t, t^{\prime} \in T^{*}(c), \max _{e \in p_{0 k(t)}} c_{e}=\max _{e \in p_{0 k\left(t^{\prime}\right)}} c_{e}$, let $p_{0 k}^{*}(c) \equiv \max _{e \in p_{0 k(t)}} c_{e}$. For $k \in N_{0}$ and $S \subset N \backslash\{k\}$, let $c^{k, S} \in \Gamma(S \cup\{k\})$ be such that $c_{0 k}^{k, S}=p_{0 k}^{*}(c)$ and $c_{i j}^{k, S}=c_{i j}$ for all $i, j \in S_{0} \cup\{k\}$, with $(i, j) \neq(0, k)$. By convention, there is no cost to the path $p_{00}$ and a problem $\left(S \cup\{0\}, c^{0, S}\right)$ is the same as $\left(S, c^{S}\right)$.

Branch Cutting: For any mest problem $(N, c)$, if there exists $S \subset N$ and $k \in N_{0} \backslash S$ such that i) for all $i \in S, i$ is a follower of $k$ in $c$, ii) for all $i \in S, j \in N \backslash(S \cup\{k\}), c_{i j} \geq \max \left\{c_{0 i}, c_{0 j}\right\}$ and iii) $c_{i i^{\prime}} \geq p_{0 k}^{*}(c)$ for all $i, i^{\prime} \in S \cup\{k\}$, then

$$
y_{i}(N, c)=\left\{\begin{array}{c}
y_{i}\left(S \cup\{k\}, c^{k, S}\right) \text { if } i \in S \\
y_{i}\left(N \backslash S, c^{N \backslash S}\right) \text { if } i \in N \backslash(S \cup\{k\})
\end{array} .\right.
$$

In words, condition i) makes sure that $S$ forms a branch that always connects to the source through $k$, condition ii) guarantees that there are no gains to connect with other agents and iii) that the costs

\footnotetext{
${ }^{1}$ With the technical condition that if the branch connects to agent $k$, we modify the direct connection cost of $k$ to the source, making it equal to the cost of the most expensive edge on its path to the source within the mcst. This is done to adequately portray the bargaining situations between the members of the branch and $k$. See the definition of the property.
} 
within the branch are no cheaper than the costs on the path from the source to $k$. Notice that if $k=\{0\}$, conditions i) and iii) impose no restrictions. Notice also that the property says nothing about the cost share of $k$, as that agent has relationships with both $S$ and $N \backslash(S \cup\{k\})$. It is however easy to find it by using Budget Balance, given that we have the shares of all other agents.

The next three properties, Full, Equal and Constant Share of Cost Reduction, are very close to each other, providing different solutions to this problem: suppose that nobody has a cheaper connection to the source than agent $i$, and that everybody can connect to him freely. Then, if the cost of connecting $i$ to the source diminishes, all else being equal, how should we split the savings? One possibility is to reward agent $i$ for the lower cost and assign him all the savings. This is particularly natural if agent $i$ took actions that resulted in the cost reduction, or if he has property rights over that edge to the source. We obtain the following property:

Full Share of Cost Reduction (FSCR): For any mest problems $(N, c),\left(N, c^{\prime}\right)$ such that $c_{0 i} \leq c_{0 j}$ and we have a free path $p_{i j}$ for all $j \in N \backslash\{i\}, c_{0 i}^{\prime}=c_{0 i}-x$ and $c_{e}^{\prime}=c_{e}$ else, then $y_{i}\left(N, c^{\prime}\right)=y_{i}(N, c)-x$ and $y_{j}\left(N, c^{\prime}\right)=y_{j}(N, c)$ for $j \in N \backslash\{i\}$, with $x \in\left[0, c_{0 i}\right]$.

At the opposite end of the spectrum, if we want to equalize shares, if agent $i$ played no special role in the cost reduction or if agent $i$ holds no property rights over his edge to the source, we can share the savings equally among all agents. We obtain the following property:

Equal Share of Cost Reduction (ESCR): For any mest problems $(N, c),\left(N, c^{\prime}\right)$ such that $c_{0 i} \leq c_{0 j}$ and we have a free path $p_{i j}$ for all $j \in N \backslash\{i\}, c_{0 i}^{\prime}=c_{0 i}-x$ and $c_{e}^{\prime}=c_{e}$ else, then $y_{j}\left(N, c^{\prime}\right)=y_{j}(N, c)-\frac{x}{|N|}$ for all $j \in N$, with $x \in\left[0, c_{0 i}\right]$.

The difference between FSCR and ESCR being mostly a difference in the way we interpret the game, it seems very natural to assume that once we settled on an interpretation of that game (even if it's on something different than the ones that inspired FSCR and ESCR), we should stick to it. Therefore, the way we deal with this kind of problems should be consistent throughout. This idea is expressed in the following property:

Constant Share of Cost Reduction (CSCR): For any mest problems $(N, c),\left(N, c^{\prime}\right)$ such that $c_{0 i} \leq c_{0 j}$ and we have a free path $p_{i j}$ for all $j \in N \backslash\{i\}, c_{0 i}^{\prime}=c_{0 i}-x$ and $c_{e}^{\prime}=c_{e}$ else, then $y_{i}\left(N, c^{\prime}\right)=y_{i}(N, c)-\frac{x}{|N|}(1+\alpha(|N|-1))$ and $y_{j}\left(N, c^{\prime}\right)=y_{j}(N, c)-\frac{x}{|N|}(1-\alpha)$ for $j \in N \backslash\{i\}$, with $x \in\left[0, c_{0 i}\right]$ and $\alpha \in \mathbb{R}$.

We can see the property as first assigning to each agent $\frac{1}{|N|}$ of the savings, before reallocating to agent $i$ a share $\alpha$ of the savings that were first assigned to the agents in $N \backslash\{i\}$. The value of $\alpha$ conveys the responsibility we assign to agent $i$ in the reduction of the cost. Two natural values of $\alpha$ are $\alpha=0$ and $\alpha=1$, corresponding respectively to ESCR and FSCR.

We now move to the familiar properties, starting with Piecewise Linearity, which says that if we can decompose a cost matrix into submatrices where the cost of all edges are ordered in the same manner as the original matrix, then the cost allocation on the original cost matrix should equal the sum of the cost allocations on the submatrices. This property (or similar versions), a weaker version than the classical Additivity property in the general setting (first proposed by Shapley (1953)), has been used in Bergantinos and Vidal-Puga (2009), Bogomolnaia and Moulin (2010), Branzei et al (2004) and Tijs et al (2006). Piecewise Linearity generates a rich class of solutions having a simple structure. Cost shares can be defined on simple elementary matrices where costs of all edges are either 0 or 1 , making it particularly appealing.

Piecewise Linearity: For any mcst problems $(N, c)$ and $\left(N, c^{\prime}\right)$, if there exists an order of the edges $\sigma: N_{0}^{p} \rightarrow\left\{1, \ldots, \frac{n(n+1)}{2}\right\}$ such that for any $e, e^{\prime} \in N_{0}^{p}$, if $\sigma(e) \leq \sigma\left(e^{\prime}\right)$, we have $c_{e} \leq c_{e^{\prime}}$ and $c_{e}^{\prime} \leq c_{e^{\prime}}^{\prime}$, then, $y\left(N, c+c^{\prime}\right)=y(N, c)+y\left(N, c^{\prime}\right)$.

Denote by $\Gamma^{e}$ the set of elementary cost matrices where all connection costs are either 0 or 1 : $\Gamma^{e}(N)=\left\{c \in \Gamma(N): c_{e} \in\{0,1\}\right.$ for all $\left.e \in N_{0}^{p}\right\}$. Let $b^{k} \in \Gamma^{e}(N)$ be such that $b_{e^{\sigma(1)}}^{k}=\ldots=b_{e^{\sigma(k-1)}}^{k}=$ 0 while $b_{e^{\sigma(k)}}^{k}=\ldots=b_{e^{\sigma(q)}}^{k}=1$.

If a solution satisfying Piecewise Linearity is well defined on $\Gamma^{e}$, it is also uniquely defined on $\Gamma$. Let $y$ be a solution defined over $\Gamma^{e}$. The piecewise linear extension of $y$ is a solution $y^{L}$ such that for all $c \in \Gamma, y^{L}(N, c)=\sum_{k=1}^{q}\left(c_{e^{\sigma(k)}}-c_{e^{\sigma(k-1)}}\right) y\left(N, b^{k}\right)$. 
The final property is the familiar Core Selection property, a stability property that guarantees that no coalition pays more than its stand-alone cost.

Core Selection: For any mcst problem $(N, c)$ and any $S \subseteq N, \sum_{i \in S} y_{i}(N, c) \leq C(S, c)$.

\section{Results}

Before stating the main results, we introduce the following notation. Let $\hat{\Gamma}^{e}$ be the set of elementary matrices where all agents have a cost of one to connect to the source and of zero to connect to another agent: $c_{0 i}=1$ and $c_{i j}=0$ for all $i, j$. Let $F(c)=\left\{i \in N \mid c_{0 i}=0\right\}$ be the set of agents having a free direct connection to the source.

We first show that a cost sharing solution satisfies Piecewise Linearity, Core Selection, Decomposition, Branch Cutting and Constant Share of Cost Reduction if and only if it is a convex combination of the folk and cycle-complete solutions.

Theorem 1 A cost sharing solution satisfies Piecewise Linearity, Core Selection, Decomposition, Branch Cutting and Constant Share of Cost Reduction if and only if it is of the form $y^{\alpha}=(1-$ $\alpha) y^{f}+\alpha y^{c c}$, where $\alpha \in[0,1]$ is the parameter in Constant Share of Cost Reduction.

Proof. Lemma A.1 in Appendix shows that the folk and cycle-complete solutions satisfy the properties. It is then obvious that any convex combination also satisfy them. We show that with Piecewise Linearity, Core Selection, Decomposition, Branch Cutting and CSCR, a cost sharing solution is uniquely defined by the value of $\alpha$ used in CSCR.

We proceed as follows: in Step 1, we show that solutions that satisfies the properties coincide for all $\hat{c} \in \hat{\Gamma}^{e}$. In Step 2, we do the same for any elementary cost matrices when $|N|=2$. Using an induction argument, we extend the result to any elementary cost matrix and any number of players in Step 3. In Step 4, we extend to any cost matrix using Piecewise Linearity. Since the values found depend on the parameter $\alpha$ used in CSCR, in Step 5 we show that the properties impose that $\alpha$ be between 0 and 1. In Step 6, we show that the remaining values are the convex combination of the folk and cycle-complete solutions.

Step 1: Show that all solutions satisfying the properties coincide for all $\hat{c} \in \hat{\Gamma}^{e}$.

Take $c \in \Gamma(N)$ such that $c_{e}=1$ for all edges. By Core Selection, $y_{i}(N, c)=1$ for all $i \in N$. By Decomposition, $y_{i}(N, c)=y_{i}(N, \hat{c})+\frac{\sum_{j \in N \backslash\{i\}} y_{i}\left(N \backslash\{j\}, c^{0 j}\right)}{|N|}$. Since for all $j \in N \backslash\{i\}, y_{i}\left(N \backslash\{j\}, c^{0 j}\right)=1$ by Core Selection, we must have that $y_{i}(N, \hat{c})=\frac{1}{|N|}$.

Step 2: Show that all solutions satisfying the properties coincide for all $c \in \Gamma^{e}(N)$ with $|N|=2$.

If $|F(c)|=0$, we can apply Decomposition and $y_{i}(N, c)=y_{i}(N, \hat{c})+\frac{\sum_{j \in N \backslash\{i\}} y_{i}\left(N \backslash\{j\}, c^{0 j}\right)}{|N|}$. Since solutions that satisfy the properties coincide for problems $(N, \hat{c}),\left(N \backslash\{j\}, c^{0 j}\right)$ (as $|N \backslash\{j\}|=1$, we must have that $y_{i}\left(N \backslash\{j\}, c^{0 j}\right)=c_{0 i}^{0 j}=c_{i j}$ by Budget Balance), they also coincide for problem $(N, c)$.

If $|F(c)|=1$ and $c_{i j}=0$, we can apply CSCR to find a unique cost allocation (for each value of $\alpha$ in that property). If $c_{i j}=1$, then by Core Selection $y_{k}(N, c)=c_{0 k}$ for all $k \in N$.

If $|F(c)|=2$, by Core Selection we must have that $y_{i}(N, c)=0$ for all $i \in N$.

Step 3: Show that all solutions satisfying the properties coincide for all $c \in \Gamma^{e}$.

Suppose now that all solutions that satisfy the properties coincide for all $c \in \Gamma^{e}(N)$ with $|N|=$ $m \geq 2$. We show that it implies that they coincide for all $c \in \Gamma^{e}(N)$ with $|N|=m+1$.

If $|F(c)|=0$, we can apply Decomposition and $y_{i}(N, c)=y_{i}(N, \hat{c})+\frac{\sum_{j \in N \backslash\{i\}} y_{i}\left(N \backslash\{j\}, c^{0 j}\right)}{|N|}$. Since solutions that satisfy the properties coincide for $(N, \hat{c}),\left(N \backslash\{j\}, c^{0 j}\right)$ (as $|N \backslash\{j\}|=m$ ), they also coincide for $(N, c)$.

If $|F(c)|=1$, say $F(c)=\{i\}$, we examine separately the cases where all or only some agents have a free path to $i$. Let $Z^{i}(c)=\left\{j \in N \backslash\{i\} \mid\right.$ there exists a free path $\left.p_{i j}\right\}$. If $Z^{i}(c)=N \backslash\{i\}$, by CSCR and our results for $|F(c)|=0$, all solutions satisfying the properties coincide (for a given value of $\alpha$ ). If $Z^{i}(c)=\emptyset$, we can apply Branch Cutting with $k=0$, and we have that $y_{i}\left(\{i\}, c^{\{i\}}\right)=y_{i}(N, c)$ and 
$y_{j}\left(N \backslash\{i\}, c^{N \backslash\{i\}}\right)=y_{j}(N, c)$ for all $j \in N \backslash\{i\}$. The solutions satisfying the properties all coincide on these problems as $|\{i\}|, \mid N \backslash\{i\}) \mid \leq m$. Otherwise, we can apply Branch Cutting and we have that $y_{j}\left(Z^{i}(c) \cup\{i\}, c^{i, Z^{i}(c)}\right)=y_{j}(N, c)$ for all $j \in Z^{i}(c)$ and $y_{j}\left(N \backslash Z^{i}(c), c^{N \backslash Z^{i}(c)}\right)=y_{j}(N, c)$ for all $j \in N \backslash\left(Z^{i}(c) \cup\{i\}\right)$. The solutions satisfying the properties all coincide on these problems as $\left|Z^{i}(c) \cup\{i\}\right|,\left|N \backslash Z^{i}(c)\right| \leq m$. By Budget Balance, $y_{i}(N, c)=C(N, c)-\sum_{j \in N \backslash\{i\}} y_{j}(N, c)$.

If $|F(c)| \geq 2$, we need to use Core Selection and Branch Cutting.

First, suppose that there exists $S \subset N$ such for $i \in S$ and $j \in N \backslash S, c_{i j} \geq \max \left(c_{0 i}, c_{0 j}\right)$. Then, we can use Branch Cutting (with $k=\{0\}$ ) and thus $y_{i}(N, c)=y_{i}\left(S, c^{S}\right)$ for all $i \in S$ and $y_{j}(N, c)=$ $y_{j}\left(N \backslash S, c^{N \backslash S}\right)$ for all $j \in N \backslash S$. The solutions satisfying the properties all coincide on these problems as $|S|,|N \backslash S| \leq m$.

For the following, we suppose that there is a free path in $N$ (that does not go through the source) between any two agents $i, j \in N$. We have two further cases:

i) We have $i \in N \backslash F(c)$ and $k \in N \backslash\{i\}$ such that $i$ is a follower of $k$ in $c$

Let $S$ be the set of all followers of $k$ in $c$. As $c$ is an elementary matrix, this implies that all free paths between the source and $i$ include agent $k$ and that $k$ has a free path to the source. Condition iii) of Branch Cutting is trivially satisfied. Condition ii) also has to be satisfied: Suppose there was an agent $l$ in $N \backslash(S \cup\{k\})$ to which an agent $m$ in $S$ had a free direct connection to. If $l$ can provide a free path to the source that does not include $k, m$ wouldn't be a follower of $k$. If $l$ doesn't provide such a path, he is also a follower of $k$. We can thus apply Branch Cutting and $y_{i}(N, c)=y_{i}\left(S \cup\{k\}, c^{k, S}\right)$ for all $i \in S$ while $y_{j}(N, c)=y_{j}\left(N \backslash S, c^{N \backslash S}\right)$ for all $j \in N \backslash S$. The solutions satisfying the properties all coincide on these problems as $|S \cup\{k\}|,|N \backslash S| \leq m$. By Budget Balance, $y_{k}(N, c)=-\sum_{i \in S} y_{i}\left(S \cup\{k\}, c^{k, S}\right)-\sum_{j \in N \backslash(S \cup\{k\})} y_{j}\left(N \backslash S, c^{N \backslash S}\right)$.

ii) For all $i \in N$, there does not exists $k \in N \backslash\{i\}$ such that $i$ is a follower of $k$ in $c$

This implies that $C(N \backslash\{i\}, c)=0$ for all $i \in N$, as no agent depends on $i$ to connect to the source at no cost. The only allocation that satisfies Core Selection is $y_{i}(N, c)=0$ for all $i \in N$.

Step 4: Show that all solutions satisfying the properties coincide for all $c \in \Gamma$.

By Steps 1-3, the solutions satisfying the properties all coincide on problems $(N, c)$ such that $c \in \Gamma^{e}$ (for a given value $\alpha$ in CSCR). By Piecewise Linearity, they also coincide for any $c \in \Gamma$.

Step 5: Show that Core Selection imposes that $\alpha \in[0,1]$.

Consider $\hat{c} \in \hat{\Gamma}^{e}$ and $c^{\prime}$ that is such that $c_{0 i}^{\prime}=0$ and $c_{e}^{\prime}=\hat{c}_{e}$ else. By the previous steps, we have that $y_{j}(N, \hat{c})=\frac{1}{|N|}$ for all $j \in N$. By CSCR, we have that $y_{i}^{\alpha}\left(N, c^{\prime}\right)=\frac{1}{|N|}-\frac{1}{|N|}(1+\alpha(|N|-1))=\frac{-\alpha(|N|-1)}{|N|}$ and $y_{j}^{\alpha}\left(N, c^{\prime}\right)=\frac{1}{|N|}-\frac{1}{|N|}(1-\alpha)=\frac{\alpha}{|N|}$ for all $j \in N \backslash\{i\}$. By Core Selection, we must have that $y_{i}^{\alpha}\left(N, c^{\prime}\right)=-\frac{\alpha(|N|-1)}{|N|} \leq 0=C\left(\{i\}, c^{\prime}\right)$, which implies that $\alpha \geq 0$. Core Selection also requires that $\sum_{j \in N \backslash\{i\}} y_{j}^{\alpha}\left(N, c^{\prime}\right)=\frac{\alpha(|N|-1)}{|N|} \leq 1=C\left(N \backslash\{i\}, c^{\prime}\right)$. It thus implies that $\alpha \leq \frac{|N|}{(|N|-1)}$. For any $\alpha>1$, there exists a number of players $K$ large enough such that $\alpha>\frac{K}{K-1}$, which implies that Core Selection is not satisfied. Lemma A.1 in Appendix shows that Core Selection is satisfied for $\alpha \in[0,1]$.

Step 6: Show that $y^{\alpha}=(1-\alpha) y^{f}+\alpha y^{c c}$.

We first show that it is true for $c \in \Gamma^{e}(N)$ with $|N|=2$. Let $N=\{i, j\}, c$ be such that $c_{0 i}=c_{0 j}=1$, $c_{i j}=0$ and $c^{\prime}$ be such that $c_{0 i}^{\prime}=0$ and $c_{e}^{\prime}=c_{e}$ else. Since $c \in \hat{\Gamma}^{e}$, we have that $y_{k}(N, c)=\frac{1}{2}$ for $k=i, j$. By CSCR, we have that $y_{i}^{\alpha}\left(N, c^{\prime}\right)=-\frac{\alpha}{2}$ and $y_{j}^{\alpha}\left(N, c^{\prime}\right)=\frac{\alpha}{2}$. It is also easy to verify that $y_{i}^{f}\left(N, c^{\prime}\right)=y_{j}^{f}\left(N, c^{\prime}\right)=0$ and $y_{k}^{\alpha}\left(N, c^{\prime}\right)=-\frac{1}{2}$ and $y_{j}^{\alpha}\left(N, c^{\prime}\right)=\frac{1}{2}$ and thus that $y^{\alpha}\left(N, c^{\prime}\right)=$ $(1-\alpha) y^{f}\left(N, c^{\prime}\right)+\alpha y^{c c}\left(N, c^{\prime}\right)$. By Step 2 above, $y^{f}(N, c)=y^{c c}(N, c)$ for any other $c \in \Gamma^{e}(N)$ with $|N|=2$, as CSCR plays no role. We thus have that $y^{\alpha}(N, c)=(1-\alpha) y^{f}(N, c)+\alpha y^{c c}(N, c)$ for all $c \in \Gamma^{e}(N)$ with $|N|=2$.

Suppose that we have shown that $y^{\alpha}(N, c)=(1-\alpha) y^{f}(N, c)+\alpha y^{c c}(N, c)$ for all $c \in \Gamma^{e}(N)$ with $|N|=m$. We show that it implies that it is also true for $c \in \Gamma^{e}(N)$ with $|N|=m+1$.

By Step 3 above, CSCR only plays a role when $F(c)=\{i\}$ and $Z^{i}(c)=N \backslash\{i\}$. Let $c$ be such a cost matrix and $c^{0 i}$ be such that $c_{0 i}^{0 i}=1$ and $c_{e}^{0 i}=c_{e}$ else. Since $\left|F\left(c^{0 i}\right)\right|=0, y^{\alpha}\left(N, c^{0 i}\right)$ is defined by cost shares on problems with less players (using Decomposition and Branch Cutting, see Step 3) for which 
we have that $y^{\alpha}=(1-\alpha) y^{f}+\alpha y^{c c}$ (by the induction argument). It is immediate that $y^{\alpha}\left(N, c^{0 i}\right)=$ $(1-\alpha) y^{f}\left(N, c^{0 i}\right)+\alpha y^{c c}\left(N, c^{0 i}\right)$. We can easily verify that $y_{i}^{\alpha}(N, c)=y_{i}^{\alpha}\left(N, c^{0 i}\right)-\frac{1}{|N|}(1+\alpha(|N|-1))$, $y_{i}^{c c}(N, c)=y_{i}^{c c}\left(N, c^{0 i}\right)-1$ and $y_{i}^{f}(N, c)=y_{i}^{f}\left(N, c^{0 i}\right)-\frac{1}{|N|}$, while $y_{j}^{\alpha}(N, c)=y_{j}^{\alpha}\left(N, c^{0 i}\right)-\frac{1}{|N|}(1-\alpha)$, $y_{j}^{c c}(N, c)=y_{j}^{c c}\left(N, c^{0 i}\right)$ and $y_{j}^{f}(N, c)=y_{j}^{f}\left(N, c^{0 i}\right)-\frac{1}{|N|}$ for all $j \in N \backslash\{i\}$. It is then obvious that $y^{\alpha}(N, c)=(1-\alpha) y^{f}(N, c)+\alpha y^{c c}(N, c)$. The result is also immediate for any other $c \in \Gamma^{e}(N)$ with $|N|=m$. The results than extends to any $c \in \Gamma$ using Piecewise Linearity.

Notice that even though CSCR puts no constraints on the value of $\alpha$ (which represents the extra share of the cost savings going to the agent who has a low cost to the source), the other properties restrict it to be between 0 and 1 , which were the two natural values we had identified. They also correspond to the Equal Share of Cost Reduction and Full Share of Cost Reduction properties respectively. Using theses properties instead of Constant Share of Cost Reduction allows us to uniquely characterize the folk and cycle-complete solutions.

Theorem 2 i) A cost sharing solution y satisfies Piecewise Linearity, Core Selection, Decomposition, Branch Cutting and Full Share of Cost Reduction if and only if it is the cycle-complete solution.

ii) A cost sharing solution y satisfies Piecewise Linearity, Core Selection, Decomposition, Branch Cutting and Equal Share of Cost Reduction if and only if it is the folk solution.

Proof. The results are immediate using Theorem 1 and Lemma A.1 in Appendix.

Independence of the properties for part i) is shown in Lemma A.2 in Appendix. However, it turns out that Piecewise Linearity, Core Selection, Decomposition, Branch Cutting and Equal Share of Cost Reduction are not independent. In fact, we can characterize the folk solution without Decomposition. To see why, consider an elementary cost matrix where $c_{0 i}=1$ for all $i \in N$ and where there exists a free path in $N$ between all agents. By Equal Share Cost of Reduction, if the cost to connect any of these agents to the source goes down to zero, all agents will see their share reduced by $\frac{1}{|N|}$. But by Core Selection, that agent cannot pay more than 0 after the change, meaning that he could not have paid more than $\frac{1}{|N|}$ before. By budget-balance, everybody must have paid $\frac{1}{|N|}$ before the change. These cost matrices are those for which Decomposition can be applied.

By contrast, with Full Share of Cost Reduction (or Constant Share of Cost Reduction with any $\alpha \in] 0,1]$ ), the agent that sees the cost of his link to the source decrease will receive a higher share of the savings than others, meaning that he could have paid more than $\frac{1}{|N|}$ before and still satisfy Core Selection. Since many different values are compatible with Core Selection, Decomposition is needed to obtain unique values.

Theorem 3 A cost sharing solution y satisfies Piecewise Linearity, Core Selection, Branch Cutting and Equal Share of Cost Reduction if and only if it is the folk solution.

Proof. Lemma A.1 in Appendix show that the folk solution satisfies the properties. We show that there is a unique solution that satisfies the properties. Let $y(N, c)$ be a solution that satisfies the properties.

Take $c \in \Gamma^{e}$ and suppose that we have $S$ such that for all $i \in S, j \in N \backslash S, c_{i j}=1$. By Branch Cutting, $y_{k}(N, c)=y_{k}\left(S, c^{S}\right)$ if $k \in S$ and $y_{k}(N, c)=y_{k}\left(N \backslash S, c^{N \backslash S}\right)$ if $k \in N \backslash S$. Therefore, in the following, we only consider $c$ such that we have a free path in $N$ between any pair of agents $i, j \in N$.

We first show that for all $c \in \Gamma^{e}$ such that $c_{0 i}=1$ for all $i \in N$ and such that there exists a free path between any agents $j, k \in N$, we have that $y_{i}(N, c)=\frac{1}{|N|}$ for all $i \in N$.

Consider $c^{j}$ to be such that $c_{0 j}^{j}=0$ and $c_{e}^{j}=c_{e}$ else. By Equal Share of Cost Reduction, $y_{i}\left(N, c^{j}\right)=$ $y_{i}(N, c)-\frac{1}{|N|}$ for all $i \in N$. Since we have that $C\left(\{j\}, c^{j}\right)=0$, we must have, by Core Selection, that $y_{j}(N, c) \leq \frac{1}{|N|}$ for all $j \in N$. Since by budget-balance we must have that $\sum_{i \in N} y_{i}(N, c)=1$, it implies that $y_{i}(N, c)=\frac{1}{|N|}$ for all $i \in N$.

By Equal Share of Cost Reduction, if $|F(c)|=1$, we have that $y_{i}(N, c)=0$ for all $i \in N$.

Finally, if $|F(c)|>1$, we need to use Core Selection and Branch Cutting. 
i) We have $i \in N \backslash F(c)$ and $k \in N \backslash\{i\}$ such that $i$ is a follower of $k$ in $c$

Let $S$ be the set of all followers of $k$ in $c$. Exactly as in Step 3i) of Theorem 1, we can apply Branch Cutting and $y_{i}(N, c)=y_{i}\left(S \cup\{k\}, c^{k, S}\right)=0$ for all $i \in S\left(\right.$ as $\left.\left|F\left(c^{k, S}\right)\right|=1\right)$ while $y_{j}(N, c)=$ $y_{j}\left(N \backslash S, c^{N \backslash S}\right)$ for all $j \in N \backslash S$. By Budget Balance, $y_{k}(N, c)=-\sum_{j \in N \backslash(S \cup\{k\})} y_{j}\left(N \backslash S, c^{N \backslash S}\right)$. We repeat the argument until we have that for all $i \in N \backslash S$, there does not exists $k \in N \backslash(S \cup\{i\})$ such that $i$ is a follower of $k$ in $c^{N \backslash S}$.

ii) For all $i \in N$, there does not exists $k \in N \backslash\{i\}$ such that $i$ is a follower of $k$ in $c$

This implies that $C(N \backslash\{i\}, c)=0$ for all $i \in N$, as no agent depends on $i$ to connect to the source at no cost. The only allocation that satisfies Core Selection is $y_{i}(N, c)=0$ for all $i \in N$.

We have shown that all solutions that satisfy the properties coincide for problems $(N, c)$ such that $c \in \Gamma^{e}$. By Piecewise Linearity, they also coincide if $c \in \Gamma$.

Independence of the properties is shown in Lemma A.3 in Appendix.

\section{Discussion}

The folk and cycle-complete solutions are Shapley values applied to different versions of the same game. It is therefore not surprising that they share many properties. Their convex combination is characterized by the properties of Piecewise Linearity, Core Selection, Decomposition, Branch Cutting and Constant Share of Cost Reduction. It is worth noting that the Kar solution, another Shapley value, satisfies all properties except Core Selection and Equal Share of Cost Reduction.

Interestingly, the characterizations of the folk solution differs from that of the cycle-complete solution only by the use of Equal Share of Cost Reduction instead of Full Share of Cost Reduction. This shows that the folk solution is fundamentally different in its approach from the cycle complete solution (as well as the Kar solution): it treats a good connection to the source as common property, with savings on its cost being shared equally by everyone who uses that connection. By opposition, the cycle-complete solution fully rewards an agent that sees the cost of its connection to the source decrease. The relevant approach to choose depends on the particular characteristic of the application being considered, notably the responsibility of the agent in that reduction in cost or in his choice of location.

While Theorem 2 offers the first characterization of the cycle-complete solutions, there has been many characterizations of the folk solutions, notably in Bergantinos and Vidal-Puga (2007, 2009) and Bogomolnaia and Moulin (2010). However, as shown in Trudeau (2012a) they all depend on the Reductionism property, that says that cost shares depend only on the irreducible matrix. The characterization proposed in Theorem 3 avoids using such a property that is very close to the method itself.

Acknowledgement 1 The author would like to thank Lars Ehlers, Gustavo Bergantinos, Juan VidalPuga and two anonymous referees for their comments. Financial support from the Social Sciences and Humanities Research Council of Canada is gladly acknowledged.

\section{References}

Bergantinos G, Vidal-Puga J (2007) A fair rule in minimum cost spanning tree problems. J. Econ. Theory 137:326-352

Bergantinos G, Vidal-Puga J (2009) Additivity in minimum cost spanning tree problems. J. Math. Econ. 45:38-42

Bird C (1976) On cost allocation for a spanning tree: A game theoretic approach. Networks 6:335-350

Bogomolnaia A, Moulin H (2010) Sharing the cost of a minimal cost spanning tree: Beyond the folk solution. Games Econ. Behav. 69:238-248 
Branzei R, Moretti S, Norde H, Tijs S (2004) The p-value for cost sharing in minimal cost spanning tree situations. Theory Decis. 56:47-61

Feltkamp V, Tijs S, Muto S (1994) On the Irreducible Core and the Equal Remaining Obligations Rule of Minimum Cost Spanning Extension Problems. Tilburg University CentER Discussion Paper 94106

Kar A (2002) Axiomatization of the Shapley value on minimum cost spanning tree games. Games Econ. Behav. 38:265-277

Kruskal J (1956) On the shortest spanning subtree of a graph and the traveling salesman problem. Proc. Am. Math. Soc. 7:48-50

Prim RC (1957) Shortest connection networks and some generalizations. Bell Syst. Tech. J. 36:13891401

Shapley LS (1953) A Value for n-person Games, in: H.W. Kuhn and A.W. Tucker (Eds), Contributions to the Theory of Games, volume II. Princeton University Press, NJ

Tijs S, Branzei R, Moretti S, Norde H (2006) Obligation rules for msct situations and their monotonicity properties. Eur. J. Oper. Res. 175:121-134

Trudeau C (2012a) Characterizations of the Kar and folk solutions for minimum cost spanning tree problems. Int. Game Theory Rev. forthcoming

Trudeau C (2012b) A new stable and more responsive cost sharing solution for mcst problems. Games Econ. Behav. 75:402-412

Trudeau C (2013) Linking the Kar and Folk Solutions Through a Problem Separation Property. University of Windsor Working Paper 1301.

\section{A Appendix}

Lemma A.1 i) The folk solution satisfies Branch Cutting, Piecewise Linearity, Core Selection, Decomposition and Equal Share of Cost Reduction, but fails Full Share of Cost Reduction.

ii) The cycle complete solution satisfies Branch Cutting, Piecewise Linearity, Core Selection, Decomposition and Full Share of Cost Reduction, but fails Equal Share of Cost Reduction.

Proof. i) Piecewise Linearity and Core Selection are well known properties of the folk solution (Trudeau (2012a)).

Branch Cutting: Suppose that there exists $S \subset N$ and $k \in N \backslash S$ such that i) for all $i \in S, i$ is a follower of $k$ in $c$, ii) for all $i \in S, j \in N \backslash(S \cup\{k\}), c_{i j} \geq \max \left\{c_{0 i}, c_{0 j}\right\}$ and iii) $c_{i i^{\prime}} \geq p_{0 k}^{*}(c)$ for all $i, i^{\prime} \in$ $S \cup\{k\}$. For all $i, j \in S \cup\{k\}$, we have that $\min _{p_{i j} \in P_{i j}\left(N_{0}\right)} \max _{e \in p_{i j}} c_{e}=\min _{p_{i j} \in P_{i j}\left((S \cup\{k\})_{0}\right)} \max _{e \in p_{i j}} c_{e}$ and thus that $\bar{c}_{i j}=\overline{c_{i j}^{k, S}}$. Similarly, we have that $\bar{c}_{i j}=\overline{c_{i j}^{N \backslash S \cup\{k\})}}$ for $i, j \in N_{0} \backslash S$. For $i \in S, \bar{c}_{0 i}=$ $\min _{p_{0 i} \in P_{0 i}\left(N_{0}\right)} \max _{e \in p_{0 i}} c_{e}$. By assumptions i) and iii), $\min _{p_{0 i} \in P_{0 i}\left(N_{0}\right)} \max _{e \in p_{0 i}} c_{e}=\min _{p_{i k} \in P_{i k}\left(S_{0} \cup\{k\}\right)} \max _{e \in p_{i k}} c_{e}=$ $\overline{c_{0 i}^{k, S}}$. For $i \in S$ and $j \in N \backslash(S \cup\{i\})$, we clearly have that $\bar{c}_{i j}=\max \left\{\bar{c}_{0 i}, \bar{c}_{0 j}\right\}$. Therefore, for any $R \subseteq S$ and $T \subseteq N \backslash(S \cup\{k\})$ we have that $C(R \cup T, \bar{c})=C(R, \bar{c})+C(T, \bar{c})$. By the properties of the Shapley value we have that $y_{i}^{f}(N, c)=\left\{\begin{array}{c}S h_{i}\left(S \cup\{k\}, \bar{c}^{S \cup\{k\}}\right)=y_{i}^{f}\left(S \cup\{k\}, c^{k, S}\right) \text { if } i \in S \\ S h_{i}\left(N \backslash S, \bar{c}^{N \backslash S}\right)=y_{i}^{f}\left(N \backslash S, c^{N \backslash S}\right) \text { if } i \in N \backslash(S \cup\{k\})\end{array}\right.$.

Decomposition: Since $c_{i j} \leq c_{0 k}$ for all $i, j, k \neq 0$, the Decomposition of the problem does not affect the computation of the irreducible matrix, as the path between $i, j \in N$ that has the cheapest most expensive edge will be the same whether we look over the edges in $N_{0}$ or $N$. Similarly, the path between $i$ and the source with the cheapest most expensive will always be such that its most expensive edge 
is the edge connecting an agent and the source. For any mest problem $(N, c)$ and any $S \subseteq N$, let $\bar{C}(S, c)=C(S, \bar{c})$.

Then, for any $i \in N$ and $S \subseteq N \backslash\{i\}$, we have that $\bar{C}\left(S, c^{0 i}\right)=\bar{C}(S \cup\{i\}, c)-a$. Therefore, for $j \in N \backslash\{i\}$, we have that

$y_{j}^{f}\left(N \backslash\{i\}, c^{0 i}\right)=\frac{\bar{C}(\{i, j\}, c)-a}{|N|-1}+\sum_{\emptyset \neq S \subseteq N \backslash\{i, j\}} \frac{|S| !(|N|-|S|-2) !}{(|N|-1) !}[\bar{C}(S \cup\{i, j\}, c)-\bar{C}(S \cup\{i\}, c)]$.

We can show that

$\sum_{i \in N \backslash\{j\}} \frac{y_{j}^{f}\left(N \backslash\{i\}, c^{0 i}\right)}{|N|}=\sum_{i \in N \backslash\{j\}} \frac{\bar{C}(\{i, j\}, c)}{(|N|-1)|N|}-\frac{a}{|N|}+\sum_{\substack{S \subseteq N \backslash\{j\} \\|S|>1}} \frac{|S| !(|N|-|S|-1) !}{|N| !}[\bar{C}(S \cup\{j\}, c)-\bar{C}(S, c)]$.

Since $\bar{C}(\{j\}, c)=C(\{j\}, c)=a$ for all $j \in N$, we have that

$$
\sum_{\substack{S \subseteq N \backslash\{j\} \\|S| \leq 1}} \frac{|S| !(|N|-|S|-1) !}{|N| !}[\bar{C}(S \cup\{j\}, c)-\bar{C}(S, c)]=\sum_{i \in N \backslash\{j\}} \frac{\bar{C}(\{i, j\}, c)}{(|N|-1)|N|}
$$

and thus that

$$
\sum_{i \in N \backslash\{j\}} \frac{y_{j}^{f}\left(N \backslash\{i\}, c^{0 i}\right)}{|N|}=-\frac{a}{|N|}+\sum_{S \subseteq N \backslash\{j\}} \frac{|S| !(|N|-|S|-1) !}{|N| !}[\bar{C}(S \cup\{j\}, c)-\bar{C}(S, c)] .
$$

As we have that $y^{f}(N, \hat{c})=\frac{a}{|N|}$,

$y_{j}^{f}(N, \hat{c})+\sum_{i \in N \backslash\{j\}} \frac{y_{j}^{f}\left(N \backslash\{i\}, c^{0 i}\right)}{|N|}=\sum_{S \subseteq N \backslash\{j\}} \frac{|S| !(|N|-|S|-1) !}{|N| !}[\bar{C}(S \cup\{j\}, c)-\bar{C}(S, c)]=y_{j}^{f}(N, c)$

ESCR: If $c_{0 i} \leq c_{0 j}$ and we have a free path $p_{i j}$ for all $j \in N \backslash\{i\}$ for all $j \in N \backslash\{i\}$ and $d_{0 i}=c_{0 i}-x$ and $d_{e}=c_{e}$ else, then $\bar{d}_{0 j}=\bar{c}_{0 j}-x$ for all $j \in N$ (as the most expensive edge on the path between 0 and $j$ is $(0, i))$. Then, $C(S, \bar{d})=C(S, \bar{c})-x$ for all $S \subseteq N$. By the properties of the Shapley value, $y_{j}^{f}(N, d)=y_{j}^{f}(N, c)-\frac{x}{|N|}$ for all $j \in N$. We can also see that it implies that FSCR is not satisfied.

ii) Piecewise Linearity: In Trudeau (2012b), the cycle-complete solution is defined using elementary matrices. Piecewise Linearity is obviously satisfied.

Branch Cutting: Suppose that there exists $S \subset N$ and $k \in N \backslash S$ such that i) for all $i \in S, i$ is a follower of $k$ in $c$, ii) for all $i \in S, j \in N \backslash(S \cup\{k\}), c_{i j} \geq \max \left\{c_{0 i}, c_{0 j}\right\}$ and iii) $c_{i i^{\prime}} \geq p_{0 k}^{*}(c)$ for all $i, i^{\prime} \in S \cup\{k\}$. If there is a cycle in $c$ that includes $i, j \in S \cup\{k\}$, that is within $S_{0}$, and for which the most expensive edge is cheaper than $c_{i j}$, the same cycle will be present in $c^{k, S}$. The same result holds for a cycle within $N \backslash S$. The only possible cycle that can involve $i \in S_{0}$ and $j \in N_{0} \backslash(S \cup\{k\})$ and such that its most expensive edge is cheaper than $c_{i j}$ has to go through the source. By assumptions ii and iii), the most expensive edge in that cycle can only be within $S \cup\{k\}$. In addition, since $c_{0 k}^{k, S}=p_{0 k}^{*}(c)$, the same cycle will be present in $c^{k, S}$, with the edge $(0, k)$ replacing (one of) the optimal path(s) $p_{0 k}$. Therefore, $c_{i j}^{*}=\left(c_{i j}^{k, S}\right)^{*}$ for all $i, j \in S_{0} \cup\{k\}$ and $c_{l m}^{*}=\left(c_{l m}^{N \backslash S}\right)^{*}$ for all $l, m \in N_{0} \backslash S$. It also implies that $c_{i j}^{*} \geq \max \left\{c_{0 i}^{*}, c_{0 j}^{*}\right\}$ for all $i \in S$ and $j \in N \backslash S$. Therefore, for any $R \subseteq S$ and $T \subseteq N \backslash(S \cup\{k\})$ we have that $C\left(R \cup T, c^{*}\right)=C\left(R, c^{*}\right)+C\left(T, c^{*}\right)$. By the properties of the Shapley value we have that $y_{i}^{c c}(N, c)=\left\{\begin{array}{c}S h_{i}\left(S \cup\{k\},\left(c^{*}\right)^{S \cup\{k\}}=y_{i}^{c c}\left(S \cup\{k\}, c^{k, S}\right) \text { if } i \in S\right. \\ S h_{i}\left(N \backslash S,\left(c^{*}\right)^{N \backslash S}=y_{i}^{c c}\left(N \backslash S, c^{N \backslash S}\right) \text { if } i \in N \backslash(S \cup\{k\})\right.\end{array}\right.$.

Decomposition: Since $c_{i j} \leq c_{0 k}$ for all $i, j, k \neq 0$, the Decomposition of the problem does not affect the computation of the cycle-complete matrix, as the cycle including $i, j \in N$ that has the cheapest 
most expensive edge will be the same whether we look over the edges in $N_{0}$ or $N$. As Decomposition only applies when $c_{0 i}=a$ and $c_{j k} \leq a$ for all $i, j, k \in N, c_{0 i}^{*}=c_{0 i}=\hat{c}_{0 i}$ for all $i \in N$. Therefore, for any $i \in N$ and $S \subseteq N \backslash\{i\}$, we have that $C^{*}\left(S, c^{0 i}\right)=C^{*}(S \cup\{i\}, c)-a$. We can apply the same procedure as for the folk solution above to show that it is satisfied.

Core Selection was shown in Trudeau (2012b).

FSCR: $c_{0 i} \leq c_{0 j}$ and we have a free path $p_{i j}$ for all $j \in N \backslash\{i\}$ and $d_{0 i}=c_{0 i}-x$ and $d_{e}=c_{e}$ else, then $c_{e}^{*}=d_{e}^{*}$ for all $e \neq(0, i)$ as there are no more or no less cycles. Then, $C\left(S \cup\{i\}, d^{*}\right)=$ $C\left(S \cup\{i\}, c^{*}\right)-x$ for all $S \subseteq N \backslash\{i\}$. By the properties of the Shapley value $y_{i}^{c c}(N, d)=y_{i}^{c c}(N, c)-x$ and $y_{j}^{c c}(N, d)=y_{j}^{c c}(N, c)$ for all $j \in N \backslash\{i\}$. We can also see that it implies that ESCR is not satisfied.

Lemma A.2 The properties of Theorem 2 i) are independent.

Proof. Let $\Gamma^{1}(N)$ be the elementary cost matrices $c$ such i) there exists a free cycle that covers all agents in $N$ and ii) $|F(c)|=0$ or 1 .

Suppose that we wish to build a solution that satisfies Piecewise Linearity, Core Selection, Branch Cutting and FSCR, and that we have already defined values for all problems $(N, c)$ such that $|N| \leq K$ and $c \in \Gamma^{e}(N)$, with $K \geq 3$.

We show that it implies unique values for all $c \in \Gamma^{e}(N) \backslash \Gamma^{1}(N)$ for all $N$ such that $|N|=K+1$.

From the proof of Theorem 1, Decomposition is used to find shares for problems $(N, c)$ when $c \in \Gamma^{e}(N)$, with $K \geq 3$

Suppose that $F(c)=\{i\}$. From the proof of Theorem 1, it is obvious that if we do not have a free path between all pair of players in $N$, we can use Branch Cutting to find the shares. That leaves us with the cases where there is a free path between all agents but not a cycle that covers $N$. Since we do not have such a cycle, we must have $S \subset N \backslash\{i\}$ such that i) for all $j \in S, j$ is a follower of $i$ in $c$, ii) for all $j \in S, k \in N \backslash(S \cup\{i\}), c_{j k}=1$. We can then apply Branch Cutting and $y_{j}(N, c)=$ $\left\{\begin{array}{c}y_{j}\left(S \cup\{i\}, c^{i, S}\right) \text { if } j \in S \\ y_{j}\left(N \backslash S, c^{N \backslash S}\right) \text { if } j \in N \backslash(S \cup\{i\})\end{array}\right.$ and, by budget balance, $y_{i}(N, c)=-\sum_{j \in N \backslash\{i\}} y_{j}(N, c)$. These cost shares are uniquely defined as $|S \cup\{i\}|,|N \backslash S| \leq K$.

Suppose that $F(c)=\emptyset$. Since we have defined unique cost shares for all cases where $|F(c)|=1$ and $c$ contains no free cycle over $N$, we can use FSCR to find unique shares for all cases where $|F(c)|=0$ and $c$ contains no free cycle over $N$.

If $|F(c)| \geq 2$, we can use the same procedure as the proof of Theorem 1 , as it does not use Decomposition. Therefore, we have defined unique values for all $c \in \Gamma^{e}(N) \backslash \Gamma^{1}(N)$ for all $N$ such that $|N|=K+1$.

To define shares over any $c$, we are left with the task to define unique shares for $c \in \Gamma^{1}(N)$, with $|N|>K$. We would have then defined unique shares for any elementary cost matrix. By Piecewise Linearity, we can then extend to any cost matrix.

Let $y^{1}(N, c)=y^{K}(N, c)$ if $|N| \geq 4$ and $c \in \Gamma^{1}(N)$ and $y^{1}(N, c)=y^{c c}(N, c)$ for any other $c \in \Gamma^{e}(N)$, $|N| \leq 4$. Use the method above to find unique cost shares for any $c \in \Gamma^{e}$. Use Piecewise Linearity to extend to any $c \in \Gamma$. We can show that $y^{1}$ satisfies Piecewise Linearity, Core Selection (as $y^{c c}$ satisfies it, and $y^{K}(N, c)$ is in the core for all $c \in \Gamma^{1}(N)$, Branch Cutting (as it cannot be applied on any $c \in$ $\Gamma^{1}(N)$ ) and FSCR (as it is satisfied by both $y^{c c}$ and $y^{K}$ ). We show that it fails Decomposition. Let $N=\{1,2,3,4\}$ and $c$ be such that $c_{12}=c_{13}=c_{14}=c_{23}=c_{34}=0$ and $c_{e}=1$ else. We have a free cycle that covers $N$ and thus $y(N, c)=y^{K}(N, c)=\left(\frac{1}{6}, \frac{1}{3}, \frac{1}{6}, \frac{1}{3}\right)$. It is easy to see that for all $i \in N$, $\left|F\left(c^{0 i}\right)\right| \geq 2$, as two or more agents are freely connected to $i$ in $c$. Therefore, $y_{j}\left(N \backslash\{i\}, c^{0 i}\right)=0$ for all $i, j \in N$. By Decomposition, we thus should have that $y(N, c)=\left(\frac{1}{4}, \frac{1}{4}, \frac{1}{4}, \frac{1}{4}\right)$.

Let $y^{2}=y^{K}$, the Kar solution. It satisfies Piecewise Linearity and fails Core Selection (Trudeau (2012a)). It is easy to show that it satisfies Decomposition, Branch Cutting and ESCR.

Let $\Gamma^{3}(N)$ be such that there exists $\{i, j, k\} \in N$ such that $c_{i j}=0, c_{0 i}=1, c_{0 j}=c_{i k}=c_{j k}=2$, $c_{0 k}=3$ and $c_{l m} \geq c_{0 l}$ for all $l \in\{i, j, k\}$ and $m \in N \backslash\{i, j, k\}$. 
Let $\Gamma^{3^{\prime}}(N)$ be such that there exists $\{i, j, k, l\} \in N$ such that $c_{j k}=0, c_{i j}=1, c_{i k}=c_{j l}=c_{k l}=2$, $c_{i l}=3$.

Build $y^{3}$ in the following manner.

i) For $c \in \Gamma(N) \backslash\left(\Gamma^{3}(N) \cup \Gamma^{3^{\prime}}(N)\right)$, let $y^{3}(N, c)=y^{c c}(N, c)$.

ii) For $c \in \Gamma^{3}(N)$ and $|N|=3$, let $y^{3}(N, c)=y^{K}(N, c)$. Notice that $y_{i}^{K}(N, c)=-\frac{1}{6}, y_{j}^{K}(N, c)=\frac{5}{6}$ and $y_{k}^{K}(N, c)=\frac{7}{3}$ and that $y^{K}(N, c) \in \operatorname{core}(N, c)$.

iii) For $c \in \Gamma^{3}(N)$ and $|N|>3$, verify if Branch Cutting can be applied. If it can, use it to define cost shares for $(N, c)$, using the cost shares defined in steps i) and ii). If not, let $y^{3}(N, c)=y^{c c}(N, c)$.

iv) For $c \in \Gamma^{3^{\prime}}(N)$ and $|N|>3$, verify if Decomposition can be applied, using the cost shares defined in steps i) through iii). (Notice that $\left.c^{0 i} \in \Gamma^{3}\right)$. If not, let $y^{3}(N, c)=y^{c c}(N, c)$.

We can show that $y^{3}$ satisfies Core Selection (as $y^{K}(N, c) \in \operatorname{core}(N, c)$ for $c \in \Gamma^{3}(N)$ and $|N|=3$, and by extension for all $c \in \Gamma^{3}$ ), Branch Cutting (by step iii) and because it does not apply for $c \in \Gamma^{3}(N)$ and $|N|=3$ ), Decomposition (by step iv) and because it does not apply for $c \in \Gamma^{3}(N)$ and $|N|=3$ ) and FSCR (as it does not apply in the matrices where $y^{3}(N, c) \neq y^{c c}(N, c)$, that is those in steps ii) through iv)). It clearly fails Piecewise Linearity.

Let $\Gamma^{4}(N)$ be the set of cost matrices $d$ such that there exists $i, j, k, l \in N$ such that $d_{0 i}=d_{i j}=$ $d_{j k}=d_{0 k}=d_{k l}=0$ and $d_{e}=1$ else. Let $\Gamma^{4}$ be the set of cost matrices in $\Gamma^{4}(N)$ for all $N$ such that $|N|=4$.

Build $y^{4}$ in the following manner:

i) Proceed with Steps 1 and 2 of the proof of Theorem 1, using FSCR instead of CSCR.

ii) Proceed with Step 3 of the proof of Theorem 1 for problems $(N, c)$ such that $|N|=3$ and $c \in \Gamma^{e}(N)$, using FSCR instead of CSCR.

iii) For problems $(N, c)$ such that $|N|=4$ and $c \in \Gamma^{e}(N) \backslash \Gamma^{4}(N)$, proceed as in Step 3 of the proof of Theorem 1, using FSCR instead of CSCR. If $c \in \Gamma 4(N)$, let $y^{4}(N, c)=(0,0,0,0)$.

iv) For problems $(N, c)$ such that $|N|>4$ and $c \in \Gamma^{e}(N)$, proceed as in Step 3 of the proof of Theorem 1, using FSCR instead of CSCR and the cost shares defined in steps i) through iii).

v) Proceed as in Step 4 of the proof of Theorem 1 to extend to any $c \in \Gamma$.

We can show that $y^{4}$ satisfies Piecewise Linearity, Core Selection, Decomposition (it does not apply for $c \in \Gamma^{4}$; for a problem $(N, c)$ such that $|N|=5$ and $|F(c)|=0$, we might have that $c^{0 i} \in \Gamma^{4}$. However, by construction, we have that $y_{i}(N, c)=\sum_{j \in N \backslash\{i\}} \frac{y_{i}\left(N \backslash\{j\}, c^{0 j}\right)}{|N|}+y_{i}(N, \hat{c})$ and thus the property is satisfied) and FSCR (it does not apply for $c \in \Gamma^{4}$; for a problem $(N, c)$ such that $|N|>5$ and $|F(c)|=1$, since the shares are defined using the shares of cost matrices where $|F(c)|=0$, which take into account the different shares for cost matrices in $\Gamma^{4}$, the property will be satisfied). Branch Cutting imposes that $y_{l}\left(\{k, l\}, c^{k,\{l\}}\right)=y_{l}(N, c)$ for any $c \in \Gamma^{4}(N)$, but we have that $y_{l}^{4}\left(\{k, l\}, c^{k,\{l\}}\right)=y_{l}^{c c}\left(\{3,4\}, c^{k,\{l\}}\right)=\frac{1}{2}$ and $y_{l}^{4}(N, c)=0$.

As shown, $y^{f}$ satisfies Piecewise Linearity, Core Selection, Decomposition and Branch Cutting but not FSCR.

The following table summarizes the findings, with "X" indicating that the property is satisfied.

$\begin{array}{cccccc} & \mathrm{D} & \mathrm{CS} & \mathrm{PL} & \mathrm{BC} & \text { FSCR } \\ y^{c c} & \mathrm{X} & \mathrm{X} & \mathrm{X} & \mathrm{X} & \mathrm{X} \\ y^{1} & & \mathrm{X} & \mathrm{X} & \mathrm{X} & \mathrm{X} \\ y^{2}=y^{K} & \mathrm{X} & & \mathrm{X} & \mathrm{X} & \mathrm{X} \\ y^{3} & \mathrm{X} & \mathrm{X} & & \mathrm{X} & \mathrm{X} \\ y^{4} & \mathrm{X} & \mathrm{X} & \mathrm{X} & & \mathrm{X} \\ y^{f} & \mathrm{X} & \mathrm{X} & \mathrm{X} & \mathrm{X} & \end{array}$

Lemma A.3 The properties of Theorem 3 are independent.

Proof. Let $\Gamma^{5}(N)$ be the set of cost matrices such that there exists $i, j, k \in N$ such that $c_{0 k}=1$ and $c_{i j}=c_{i k}=c_{j k}=c_{0 i}=c_{0 j}=0$.

Build $y^{5}$ in the following manner. 
i) For any $c \in \Gamma^{e}(N) \backslash \Gamma^{5}(N)$ such that $|N| \leq 3$, let $y^{5}(N, c)=y^{f}(N, c)$.

ii) For any $c \in \Gamma^{5}(N)$ such that $|N|=3$, let $y^{5}(N, c)=y^{K}(N, c)$, which is such that $y_{i}=y_{j}=-\frac{1}{6}$ and $y_{k}=\frac{1}{3}$.

iii) Suppose that we have defined cost shares for all $c \in \Gamma^{e}(N)$ such that $|N| \leq M$. We define cost shares for all $c \in \Gamma^{e}(N)$ such that $|N|=M+1$ in the following manner. If $F(c) \leq 1$, we proceed as in the proof of Theorem 3. If $F(c) \geq 2$, we first apply, if possible, Branch Cutting to connect the shares to those defined in $\Gamma^{e}(N)$ such that $|N| \leq M$. If we cannot apply Branch Cutting, we proceed as in the proof of Theorem 3 .

iv) We apply Piecewise Linearity to define shares for any $c \in \Gamma$.

By construction, $y^{5}$ satisfies Piecewise Linearity, Branch Cutting (by step iii) and because Branch Cutting does not apply on $c \in \Gamma^{5}(N)$ such that $|N|=3$ ) and ESCR (because $y^{f}$ satisfies ESCR and it does not apply on any $c \in \Gamma^{5}(N)$ ). It does not satisfy Core Selection as if $N=\{1,2,3\}$ and $c \in \Gamma^{5}(N)$, the only core allocation is $(0,0,0)$.

Let $\Gamma^{6}(N)$ be such that there exists $\{i, j, k\} \in N$ such that $c_{i j}=0, c_{0 i}=1, c_{0 j}=c_{i k}=c_{j k}=2$, $c_{0 k}=3$ and $c_{l m} \geq c_{0 l}$ for all $l \in\{i, j, k\}$ and $m \in N \backslash\{i, j, k\}$.

Build $y^{6}$ in the following manner.

i) For $c \in \Gamma(N) \backslash \Gamma^{6}(N)$, let $y^{6}(N, c)=y^{f}(N, c)$.

ii) For $c \in \Gamma^{6}(N)$ and $|N|=3$, let $y^{6}(N, c)=y^{K}(N, c)$. Notice that $y_{i}^{K}(N, c)=-\frac{1}{6}, y_{j}^{K}(N, c)=\frac{5}{6}$ and $y_{k}^{K}(N, c)=\frac{7}{3}$ and that $y^{K}(N, c) \in \operatorname{core}(N, c)$.

iii) For $c \in \Gamma^{6}(N)$ and $|N|>3$, verify if Branch Cutting can be applied. If it can, use it to define cost shares for $(N, c)$, using the cost shares defined in steps i) and ii). If not, let $y^{6}(N, c)=y^{f}(N, c)$.

We can show that $y^{6}$ satisfies Core Selection (as $y^{K}(N, c) \in \operatorname{core}(N, c)$ for $c \in \Gamma^{6}(N)$ and $|N|=3$, and by extension for all $c \in \Gamma^{6}$ ), Branch Cutting (by step iii) and because it does not apply for $c \in \Gamma^{6}(N)$ and $|N|=3$ ) and ESCR (as it does not apply in the matrices where $y^{6}(N, c) \neq y^{f}(N, c)$, that is those in steps ii) and iii)). It clearly fails Piecewise Linearity.

Let $\Gamma^{7}(N)$ be the set of cost matrices $d$ such that there exists $i, j, k, l \in N$ such that $d_{0 i}=d_{i j}=$ $d_{j k}=d_{0 k}=d_{k l}=0$ and $d_{e}=1$ else. Let $\Gamma^{7}$ be the set of cost matrices in $\Gamma^{7}(N)$ for all $N$ such that $|N|=4$.

Build $y^{7}$ in the following manner:

i) Proceed with Steps 1 and 2 of the proof of Theorem 1, using ESCR instead of CSCR.

ii) Proceed with Step 3 of the proof of Theorem 1 for problems $(N, c)$ such that $|N|=3$ and $c \in \Gamma^{e}(N)$, using ESCR instead of CSCR.

iii) For problems $(N, c)$ such that $|N|=4$ and $c \in \Gamma^{e}(N) \backslash \Gamma^{7}(N)$, proceed as in Step 3 of the proof of Theorem 1, using ESCR instead of CSCR. If $c \in \Gamma^{7}(N)$, let $y_{i}^{7}(N, c)=y_{j}^{7}(N, c)=0, y_{k}^{7}(N, c)=-\frac{1}{2}$ and $y_{l}^{7}(N, c)=\frac{1}{2}$.

iv) For problems $(N, c)$ such that $|N|>4$ and $c \in \Gamma^{e}(N)$, proceed as in Step 3 of the proof of Theorem 1, using ESCR instead of CSCR and the cost shares defined in steps i) through iii).

v) Proceed as in Step 4 of the proof of Theorem 1 to extend to any $c \in \Gamma$.

We can show that $y^{7}$ satisfies Piecewise Linearity, Core Selection (as $y^{7} \in \operatorname{core}(N, c)$ for all $c \in \Gamma^{7}$ ) and ESCR (it does not apply for $c \in \Gamma^{7}$; for a problem $(N, c)$ such that $|N|>4$ and $|F(c)|=1$, since the shares are defined using the shares of cost matrices where $|F(c)|=0$, which take into account the different shares for cost matrices in $\Gamma^{7}$, the property will be satisfied). Branch Cutting imposes that $y_{l}\left(\{k, l\}, c^{k,\{l\}}\right)=y_{l}(N, c)$ for any $c \in \Gamma^{7}(N)$, but we have that $y_{l}^{7}\left(\{k, l\}, c^{k,\{l\}}\right)=y_{l}^{f}\left(\{3,4\}, c^{k,\{l\}}\right)=0$ and $y_{l}^{7}(N, c)=\frac{1}{2}$.

As shown, $y^{c c}$ satisfies Piecewise Linearity, Core Selection, Branch Cutting but not ESCR.

The following table summarizes the findings, with " $\mathrm{X}$ " indicating that the property is satisfied. 


$\begin{array}{ccccc} & \mathrm{CS} & \mathrm{PL} & \mathrm{BC} & \mathrm{ESCR} \\ y^{f} & \mathrm{X} & \mathrm{X} & \mathrm{X} & \mathrm{X} \\ y^{5} & & \mathrm{X} & \mathrm{X} & \mathrm{X} \\ y^{6} & \mathrm{X} & & \mathrm{X} & \mathrm{X} \\ y^{7} & \mathrm{X} & \mathrm{X} & & \mathrm{X} \\ y^{c c} & \mathrm{X} & \mathrm{X} & \mathrm{X} & \end{array}$

